\title{
An Introduction to Newer PET Diagnostic Agents and Related Therapeutic Radiopharmaceuticals
}

\author{
Ellie Mantel, CNMT, RT(N), FSNMMITS ${ }^{1}$ and Jessica Williams, CNMT, RT(N), FSNMMITS ${ }^{2}$ \\ ${ }^{I}$ Department of Radiology, Hospital of the University of Pennsylvania, Philadelphia, Pennsylvania; and ${ }^{2}$ Molecular Imaging \\ Department, Harley Street Clinic, HCA Healthcare, London, England
}

\begin{abstract}
CE credit: For CE credit, you can access the test for this article, as well as additional JNMT CE tests, online at https://www.snmmilearningcenter.org. Complete the test online no later than September 2022. Your online test will be scored immediately. You may make 3 attempts to pass the test and must answer $80 \%$ of the questions correctly to receive $1.0 \mathrm{CEH}$ (Continuing Education Hour) credit. SNMMI members will have their CEH credit added to their VOICE transcript automatically; nonmembers will be able to print out a CE certificate upon successfully completing the test. The online test is free to SNMMI members; nonmembers must pay $\$ 15.00$ by credit card when logging onto the website to take the test.
\end{abstract}

With new radiopharmaceuticals constantly in development, the field of nuclear medicine is ever evolving. This continuing education article reviews some of the newer, less frequently used radiopharmaceuticals for PET diagnostic imaging and related therapeutic protocols. The radiopharmaceuticals discussed include U.S. Food and Drug Administration-approved tracers as well as tracers that are in clinical trials and have yet to obtain Food and Drug Administration approval. This article contains straightforward information on each tracer discussed, including disease process imaged or treated, half-life, energy, primary uses, basic protocols, distribution, and side effects. Although not all tracers may be available in rural locations, this information can be helpful in determining future patient-care offerings.

Key Words: neuroendocrine tumor; prostate cancer; Alzheimer disease; amyloid imaging; cardiovascular imaging; neurologic PET imaging

J Nucl Med Technol 2019; 47:203-209

DOI: $10.2967 /$ jnmt.118.224022

\section{A} nuclear medicine and molecular imaging continue to grow and expand, so does the number of radiopharmaceuticals that are available in our imaging and therapeutic arsenal. Numerous new radiopharmaceutical tracers have been developed for diagnostic PET and therapeutic applications. Not all radiopharmaceuticals discussed in this article are Food and Drug Administration (FDA)-approved; however, most are approved but may be geographically limited in availability. For radiopharmaceuticals still in the clinical trial phase, specific patient preparation and

\footnotetext{
Received May 21, 2018; revision accepted Feb. 20, 2019.

For correspondence or reprints contact: Ellie Mantel, CNMT, RT(N), FSNMMI-TS, Hospital of the University of Pennsylvania, 745 Valley Ave., Hammonton, NJ 08037.

E-mail: eleanor.mantel@uphs.upenn.edu

Published online Apr. 24, 2019.

COPYRIGHT @ 2019 by the Society of Nuclear Medicine and Molecular Imaging.
}

imaging protocols have yet to be finalized. The objective of this paper is to provide an overview of newer radiopharmaceuticals and their uses related to neuroendocrine tumors (NETs), prostate cancer, Alzheimer disease, and coronary artery disease.

\section{RADIOPHARMACEUTICALS FOR NETS}

The neuroendocrine system comprises specialized nerve cells that receive messages from the nervous system and then produce hormones (1). These hormones control many body functions. NETs are relatively rare tumors, with fewer than 200,000 reported cases in the United States annually. They do not consume glucose and generally manifest as an overexpression of the hormone somatostatin or norepinephrine (2).

Not all NETs are malignant; some are benign, but the malignant tumors can metastasize. NETs are slow-growing tumors occurring in a variety of locations within the neuroendocrine system. For example, carcinoid tumors, which can form in many areas of the body, most commonly are found in the digestive system, including the stomach, small intestine, appendix, and rectum. NETs can also develop in the lungs and thymus, and although it is less likely, they can develop in the pancreas, ovaries, testicles, and kidneys. Because NETs can occur in such varied locations, they are not typically diagnosed until they reach advanced stages.

There are multiple types of NETs, including pheochromocytoma, medullary carcinoma, gastrinoma, insulinoma, pancreatic NET, and paraganglioma, to name a few. Depending on the type of tumor, some NETs also secrete hormones, with symptoms varying depending on the type of NET.

Several somatostatin analog tracers have been labeled with ${ }^{68} \mathrm{Ga}$ and used for imaging, including DOTATATE, DOTATOC, and DOTANATE. DOTATATE has been in use in Europe for over $10 \mathrm{y}$; however, it only recently received approval in the United States from the FDA in 2016.

DOTATATE, DOTATOC, and DOTANATE are somatostatin receptor peptides primarily used to determine whether a patient's tumor is somatostatin receptor-positive. Determining 
somatostatin receptor status is essential for treatment planning because not all NETs are somatostatin receptor-positive. Patients with somatostatin receptor-positive NETs are ideal candidates for ${ }^{177} \mathrm{Lu}$ or ${ }^{90} \mathrm{Y}$-DOTA somatostatin ana$\log$ therapies.

In addition to determining the receptor status of the tumor, somatostatin analog imaging aids physicians in identifying the presence and extent of metastatic disease throughout the body. Diagnostic doses are also used to monitor a patient's response to therapy or disease progression (3).

\section{${ }^{68} \mathrm{Ga}-\mathrm{DOTATATE}$ Imaging}

${ }^{68} \mathrm{Ga}$-DOTATATE (NETSPOT; Advanced Accelerator Applications) is a radioactive diagnostic agent that is approved for the localization of somatostatin receptor-positive NETs. ${ }^{68} \mathrm{Ga}$ is generator-produced from ${ }^{68} \mathrm{Ge}$, which has a half-life of $270 \mathrm{~d}$ (4). This long half-life allows a nuclear pharmacy to use the generator for 6-12 mo depending on the number of elutions. The expiratory limit is approximately 550 elutions (recently increased from the previously reported 400 total elutions). Total elutions include patient doses as well as the weekly quality control elution. Quality control on the generator eluate is performed weekly to determine germanium breakthrough. Additionally, quality control of the generator eluate must also be performed if the generator has been moved or if more than $72 \mathrm{~h}$ have elapsed between elutions. The generator can be eluted up to 3 times each day at intervals of $4 \mathrm{~h}$ to allow for a $90 \%$ maximum yield.

${ }^{68} \mathrm{Ga}$ has a half-life of $68 \mathrm{~min}$ and decays by positron emission to stable ${ }^{68} \mathrm{Zn}$. Because of its short half-life, coordination between the imaging site and the nuclear pharmacy preparing the ${ }^{68} \mathrm{Ga}$-DOTATATE kit is essential. Additionally, a rural institution's geographic location may be a limiting factor in performing ${ }^{68} \mathrm{Ga}$-DOTATATE imaging.

The recommended adult and pediatric dose is weightbased at $2 \mathrm{MBq} / \mathrm{kg}(0.054 \mathrm{mCi} / \mathrm{kg})$, with a maximum dose of $200 \mathrm{MBq}(5.4 \mathrm{mCi})$ administered via intravenous injection $(4,5)$. No specific patient preparation is required with the exception of discontinuing short-acting somatostatin analogs (e.g., octreotide) $24 \mathrm{~h}$ before ${ }^{68} \mathrm{Ga}$-DOTATATE administration. Imaging is performed 40-90 min after administration of ${ }^{68} \mathrm{Ga}$-DOTATATE. For PET/CT imaging, oral barium contrast medium may be administered at the time of injection. Images are acquired from skull to mid thigh with the patient supine.

Normal distribution of ${ }^{68} \mathrm{Ga}$-DOTATATE includes liver, kidneys, bladder, bowel, spleen, pituitary, salivary glands, thyroid, and adrenal medulla (Fig. 1). The spleen and the urinary bladder wall receive the highest absorbed dose. Approximately $12 \%$ of the injected dose will be excreted in the urine within $4 \mathrm{~h}$ of dose administration.

As would typically be expected, the resolution of the ${ }^{68} \mathrm{Ga}$-DOTATATE PET scan is better than that of traditional

${ }^{111}$ In-diethylenetriaminepentaacetic acid (octreotide) planar imaging. However, ${ }^{68} \mathrm{Ga}$-DOTATATE imaging will not completely replace ${ }^{111} \mathrm{In}$-octreotide for 2 reasons. First, many

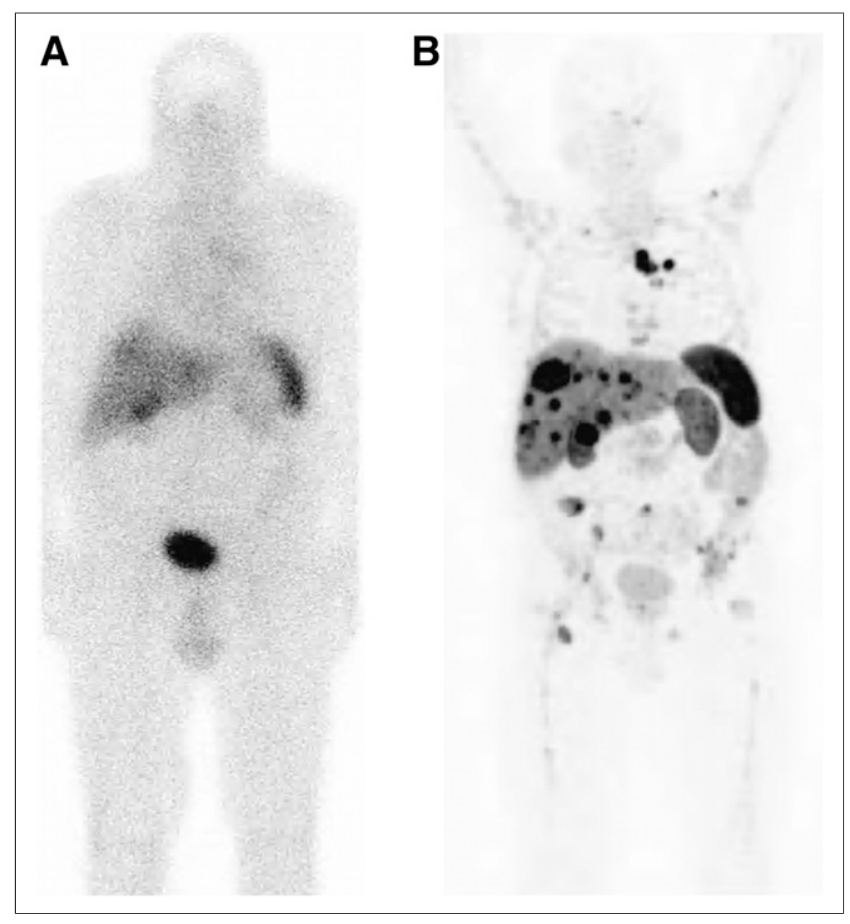

FIGURE 1. Two images from same patient obtained $3 \mathrm{wk}$ apart: planar ${ }^{111} \mathrm{In}$-octreotide scan (A) and ${ }^{68} \mathrm{Ga}$-DOTATATE PET scan (B). Lesions within liver are more easily identified on higher resolution of PET scan than on planar octreotide scan.

nuclear imaging departments do not have PET equipment, and second, access to ${ }^{68} \mathrm{Ga}$-DOTATATE is geographically constrained to areas close to a nuclear pharmacy.

\section{Lu-DOTATATE Therapy}

${ }^{177}$ Lu-DOTATATE (Lutathera; Advanced Accelerator Applications) is a reactor-produced therapeutic radiopharmaceutical for the treatment of NETs (6). ${ }^{177} \mathrm{Lu}$ has ideal physical properties for use in cancer treatment, including a particle range of $1.5 \mathrm{~mm}$, a half-life of $6.73 \mathrm{~d}$, and being a $\beta$-emitter (490 keV) and $\gamma$-emitter (113 keV and $210 \mathrm{keV}$ ), allowing for both treatment and imaging (7). Although this radiopharmaceutical has been used in Europe for more than $10 \mathrm{y}$, it only recently received FDA approval in January 2018. Peptide receptor radionuclide therapy is a cancer treatment that uses the characteristics of a cancer cell to deliver the radiation. A group of amino acids called peptides is sometimes found on the surface of the cell, and these peptides have the ability to bind to certain hormones. In peptide receptor radionuclide therapy, the receptor on the surface of the cancer cell binds to a peptide, which is paired with a radionuclide. This combination of peptide and radionuclide is called a radiopeptide. The radiopeptide is then administered intravenously and seeks cancer cells' somatostatin receptors. After attaching to the cancer cells, the radiopeptide delivers radiation directly to the cells $(8)$.

${ }^{177} \mathrm{Lu}$-DOTATATE is a peptide receptor radionuclide therapy used for patients when surgery is not an option. Patients must have NETs that are somatostatin receptor-positive for this treatment to be an option. 
The recommended dose of ${ }^{177} \mathrm{Lu}$-DOTATATE is 7,400 MBq (200 mCi) every 8 wk (9). The therapy regimen consists of a total of 4 doses. This treatment is administered via slow intravenous infusion in conjunction with an infusion of amino acids, over several hours. The amino acids are administered before, during, and after the ${ }^{177} \mathrm{Lu}$-DOTATATE dose to protect against renal toxicity. Somatostatin and its analogs competitively bind to somatostatin receptors and may interfere with the efficacy of ${ }^{177} \mathrm{Lu}$-DOTATATE. Patient preparation includes discontinuation of long-acting somatostatin analogs for at least $4 \mathrm{wk}$ and short-acting octreotide at least $24 \mathrm{~h}$ before each ${ }^{177} \mathrm{Lu}$-DOTATATE dose. The infusion procedure takes $6-8 \mathrm{~h}$ to complete. On completion of the therapy administration, patients are discharged with standard radiation precautions equivalent to those received by a patient who undergoes ${ }^{131}$ I-sodium iodide thyroid therapy.

The patient's red blood cell, white blood cell, and platelet counts must be monitored after treatment, and subsequent treatment doses may be reduced or terminated if myelosuppression occurs $(6)$. The most common reactions to this therapy include lymphopenia, increased $\gamma$-glutamyl transferase level, vomiting, nausea, increased aspartate aminotransferase level, increased alanine aminotransferase level, hyperglycemia, and hypokalemia (9). Patients may be pretreated with antiemetics to minimize or eliminate episodes of nausea and vomiting. Other less commonly reported side effects are myelodysplastic syndrome and leukemia.

Given that ${ }^{177} \mathrm{Lu}$-DOTATATE is a $\gamma$-emitter, posttherapy scans and dosimetry can be performed. These can be beneficial in providing additional information, including the intensity of uptake and the localization of the ${ }^{177} \mathrm{Lu}$-DOTATATE. Dosimetry is generally performed during the first round of treatment. Actual protocols will depend on the parameters of the clinical imaging center.

\section{RADIOPHARMACEUTICALS FOR PROSTATE CANCER}

Prostate cancer is the second most common cancer in American men, preceded only by skin cancer. In 2018, approximately 165,000 new cases were diagnosed, and approximately 29,000 deaths were attributed to prostate cancer (10). These numbers equate to approximately 1 in 7 men being diagnosed. It is rare for a man to be diagnosed before the age of 40 , and 6 in 10 cases diagnosed will be men over 65 y old. Some symptoms of prostate cancer include problems urinating; blood in urine or semen; erectile dysfunction; pain in hips, back, or ribs (due to metastasis); and weakness or numbness in legs or feet.

Until recently, the diagnostic imaging agents for evaluating recurrent prostate cancer in men were limited. Currently, there are several new radiopharmaceuticals for diagnosing recurrent prostate cancer that are either FDA-approved or in clinical trials. ${ }^{18} \mathrm{~F}$-fluciclovine, ${ }^{11} \mathrm{C}$-choline ${ }^{18} \mathrm{~F}$-choline, ${ }^{11} \mathrm{C}$-acetate, and ${ }^{68} \mathrm{Ga}$-prostate specific membrane antigen (PSMA) are all useful PET agents for imaging these patients. At the time of publishing, ${ }^{18} \mathrm{~F}$-fluciclovine is the only FDA-approved radionuclide. ${ }^{177} \mathrm{Lu}$-PSMA is currently being investigated and has shown promise as a therapeutic agent.

\section{${ }^{18}$ F-Fluciclovine}

In 2016, the FDA approved ${ }^{18} \mathrm{~F}$-fluciclovine (Axumin; Blue Earth Diagnostics) for imaging to detect recurrent prostate cancer in men with a previous history of prostatectomy and a rising prostate-specific antigen level after treatment (11). ${ }^{18} \mathrm{~F}$-fluciclovine is an amino acid analog (12). Amino acids are useful in cancer imaging because they are needed for cell metabolism and growth. ${ }^{18} \mathrm{~F}$-fluciclovine has a 110-min half-life.

The recommended imaging dose of ${ }^{18} \mathrm{~F}$-fluciclovine is $370 \mathrm{MBq}(10 \mathrm{mCi})$ administered as a bolus intravenous injection $(11,12)$. Patient preparation includes avoiding strenuous exercise the day before the study and limiting liquids for at least $4 \mathrm{~h}$ before the dose administration. To avoid potential early bladder activity from the ${ }^{18} \mathrm{~F}$-fluciclovine, it is recommended that the patient not void immediately before imaging (12). After the administration of ${ }^{18} \mathrm{~F}$-fluciclovine and a 3- to 5-min delay to allow for bloodpool clearance, the patient is imaged supine with the arms above the head. Images begin at the level of the pelvis and move to the skull base. It is important to image the pelvis first because although initial bladder uptake is minimal, it can increase over time.

Normal distribution of ${ }^{18} \mathrm{~F}$-fluciclovine includes the liver, pancreas, pituitary gland, salivary glands, lymphoid tissue of the Waldeyer ring, thyroid gland, breast parenchyma, esophagus, stomach, bowel, and renal parenchyma, with the pancreas and heart wall receiving the highest absorbed radiation dose (Fig. 2) (11,12). Excretion of the radiopharmaceutical is via urine, with $3 \%$ excreted at $4 \mathrm{~h}$ and up to $5 \%$ at $24 \mathrm{~h}$. Prostate cancer recurrence is diagnosed by comparing abnormal ${ }^{18} \mathrm{~F}$-fluciclovine uptake with tissue background.

\section{${ }^{11} \mathrm{C}$-Choline}

Another option for imaging recurrent prostate cancer is ${ }^{11} \mathrm{C}$-choline. Choline is an important nutrient used in cell membrane synthesis and phospholipid metabolism. A higher intake or status of choline in plasma and tissues

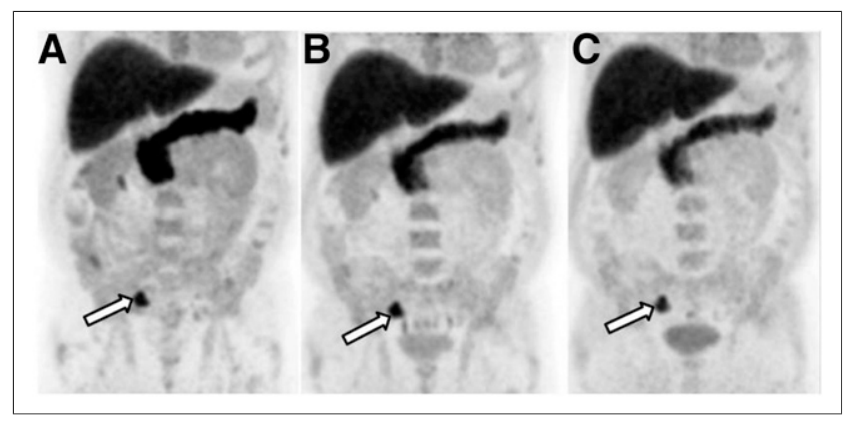

FIGURE 2. Abnormal uptake (arrows) of ${ }^{18} \mathrm{~F}$-fluciclovine in metastatic lymph node in right pelvis on PET images obtained 5-16 min after injection (A), 17-28 min after injection (B), and 29-40 min after injection (C). Uptake increases in urinary bladder over time. 
has been related to higher cancer risk. Prostate cancer shows elevated levels of ${ }^{11} \mathrm{C}$-choline uptake and levels of certain choline metabolites. Choline metabolites can be used as potential prognostic biomarkers for the management of prostate cancer patients. Increased ${ }^{11} \mathrm{C}$-choline uptake is seen in prostate tumors $(13) .{ }^{11} \mathrm{C}$-choline for imaging is cyclotronproduced, with a 20-min half-life (14). This short half-life can be a limiting factor in an institution's ability to image using ${ }^{11} \mathrm{C}$-choline, because a cyclotron must be close to the facility.

The recommended dose of ${ }^{11} \mathrm{C}$-choline is $370-740 \mathrm{MBq}$ $(10-20 \mathrm{mCi})$. Patient preparation includes taking nothing by mouth for $6 \mathrm{~h}$ before injection to decrease excretion into the bowel. The agent is administered intravenously with the patient lying supine on the imaging table (14). Intravenous hydration is recommended after dose administration to prevent pooling of the radiopharmaceutical in the kidneys (15). Imaging begins immediately after the injection. The patient is scanned from the base of the pelvis to the base of the skull. On completion of the initial imaging, the patient is asked to void before the acquisition of additional images of the pelvic region. The acquisition time of the additional pelvis images must be increased to account for the decay of ${ }^{11} \mathrm{C}$.

Normal distribution of ${ }^{11} \mathrm{C}$-choline is in the pancreas, liver, spleen, kidneys, lacrimal glands, salivary glands, and bowel, with little urinary excretion during the imaging time frame (Fig. 3). Uptake in bone marrow may also be present. Because of the excretion mechanism, the kidneys, ureters, and bladder may be visualized (15). The pancreas and kidneys receive the highest absorbed dose (14). Less than $2 \%$ of ${ }^{11} \mathrm{C}$ is excreted in the urine $1.5 \mathrm{~h}$ after injection. Prostate cancer recurrence is diagnosed by comparing ${ }^{11} \mathrm{C}$ choline uptake with tissue background.

A potential alternative to ${ }^{11} \mathrm{C}$-choline is ${ }^{18} \mathrm{~F}$-choline. ${ }^{18} \mathrm{~F}$ choline is currently being researched for imaging prostate cancer for staging, restaging, and monitoring treatment response. Although there are not enough data to support its

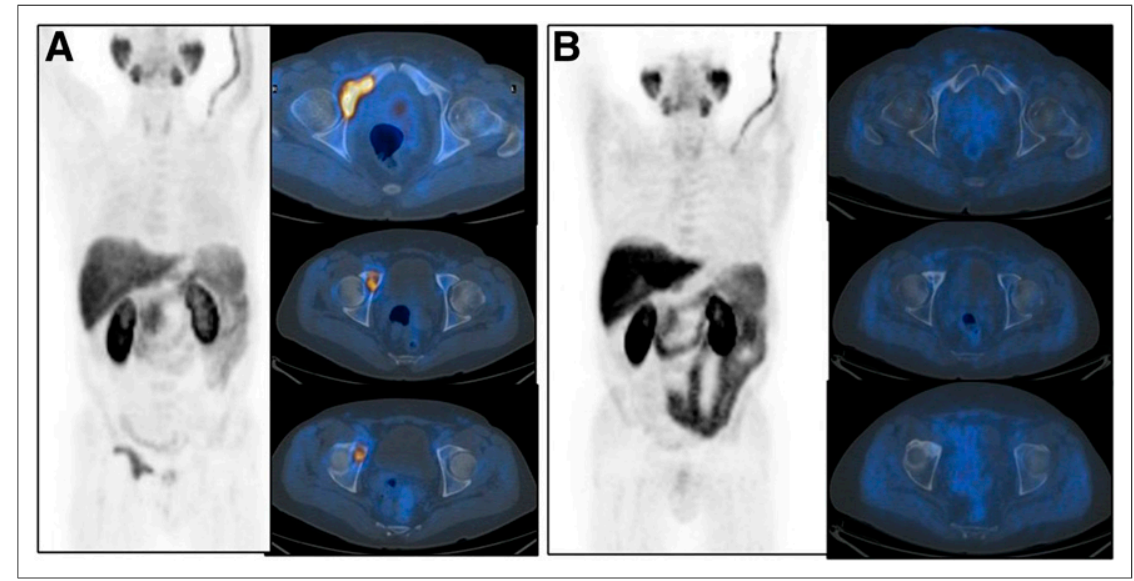

FIGURE 3. Abnormal uptake of ${ }^{11} \mathrm{C}$-choline in right ilium and pubis on PET (left panels in $A$ and $B$ ) and PET/CT (right panels in $A$ and $B$ ) images obtained before $(A)$ and after $(B)$ treatment. use for primary prostate cancer detection, it has been used to image patients with rising prostate-specific antigen levels and repeated negative biopsy results (16). It may also play a role in salvage therapy. The sensitivity and specificity for evaluating bone metastasis are high. Normal uptake of ${ }^{18} \mathrm{~F}$ choline is seen in the pancreas, liver, spleen, and kidneys (17). Uptake in the bowel varies, and ${ }^{18} \mathrm{~F}$-choline is excreted in the urine.

\section{${ }^{11} \mathrm{C}$-Acetate}

${ }^{11} \mathrm{C}$-acetate is another tracer used for imaging recurrent prostate cancer in patients with rising prostate-specific antigen levels who have had a prostatectomy and previous therapy (18). There are several clinical trials in North America, Europe, and Southeast Asia evaluating its use in prostate cancer imaging and in other disease processes.

As previously mentioned, ${ }^{11} \mathrm{C}$ is cyclotron-produced with a 20-min half-life. Again, because of its short half-life, a cyclotron must be near the imaging facility. Prostate imaging is performed as a dynamic scan. The typical dose administered is $5 \mathrm{MBq} / \mathrm{kg}(0.135 \mathrm{mCi} / \mathrm{kg})(18)$. Patient preparation has not yet been finalized because the clinical trials are still ongoing.

Initially, ${ }^{11} \mathrm{C}$-acetate uptake is visualized in the heart, kidneys, lungs, liver, spleen, pancreas, salivary glands, stomach, and bowel (Fig. 4). As time progresses, activity in the heart and kidneys diminishes. However, activity in the salivary glands, pancreas, liver, spleen, and bowel remains. The pancreas receives the highest absorbed radiation dose.

\section{${ }^{68} \mathrm{Ga}-\mathrm{PSMA}$}

PSMA is a transmembrane protein present in prostatic tissues (19). Elevated PSMA expression is seen in several malignancies but is most notably seen in prostate cancer. Levels of PSMA expression can help determine disease prognosis. For noninvasive imaging, PSMA is tagged to ${ }^{68} \mathrm{Ga}$ to help localize the site of recurrence in patients with prostate-specific antigen values between 0.2 and $10 \mathrm{ng} / \mathrm{mL}$ (19). The results from a ${ }^{68} \mathrm{Ga}$-PSMA scan can also help guide salvage therapy.

The recommended dose for imaging is weight-based, at $1.8-2.2 \mathrm{MBq} / \mathrm{kg}$ $(0.049-0.060 \mathrm{mCi} / \mathrm{kg})$. No patient preparation is required for the study; however, the patient should be well hydrated $2 \mathrm{~h}$ before dose administration. The dose is administered via an intravenous bolus injection. The uptake period is typically $60 \mathrm{~min}$, with a range of 50-100 min acceptable. The primary route of excretion is via the urinary system; therefore, the patient should void immediately before imaging. Additionally, because a small amount of activity can be cleared via the hepatobiliary system, caution and careful consideration should be taken during the 


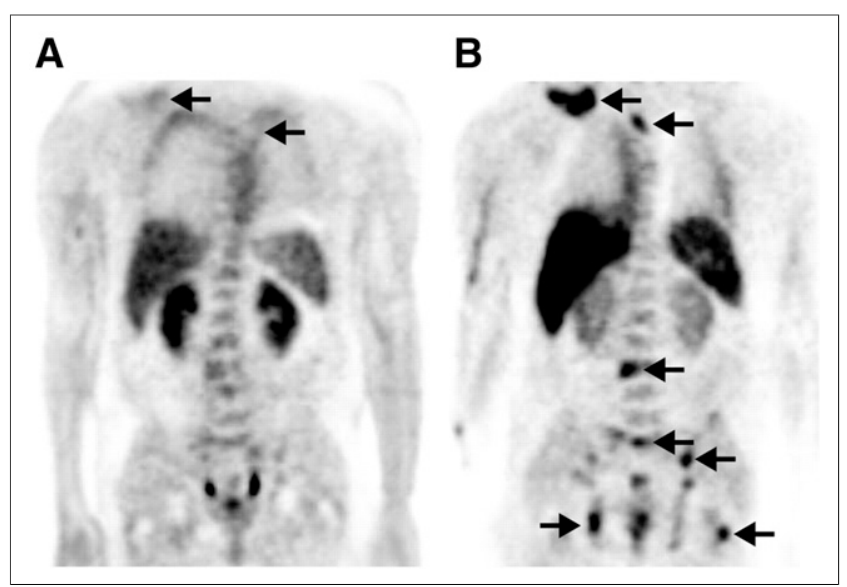

FIGURE 4. ${ }^{18} \mathrm{~F}-\mathrm{FDG}(\mathrm{A})$ and ${ }^{11} \mathrm{C}$-acetate $(\mathrm{B})$ uptake (arrows) on PET images of patient with metastatic prostate cancer.

interpretation of the study to avoid confusing this activity with possible recurrence. Scanning typically begins at the level of the mid thigh and proceeds upward to the head. In the event of activity retention in the ureters, $20 \mathrm{mg}$ of furosemide may be given intravenously to patients who have no contraindications to furosemide. This step may be useful in reducing the incidence of false-positive readings.

Normal biodistribution includes the lacrimal glands, salivary glands, liver, spleen, small intestine, colon, and kidneys (Fig. 5).

\section{${ }^{177}$ Lu-PSMA}

Although ${ }^{68} \mathrm{Ga}$-PSMA is a tracer for imaging prostate cancer, there is research being performed with ${ }^{177} \mathrm{Lu}$-PSMA as a therapeutic option (7). ${ }^{177} \mathrm{Lu}$-PSMA is a reactor-produced $\beta$-therapy being researched in the United States for treating patients with metastatic prostate cancer or prostate

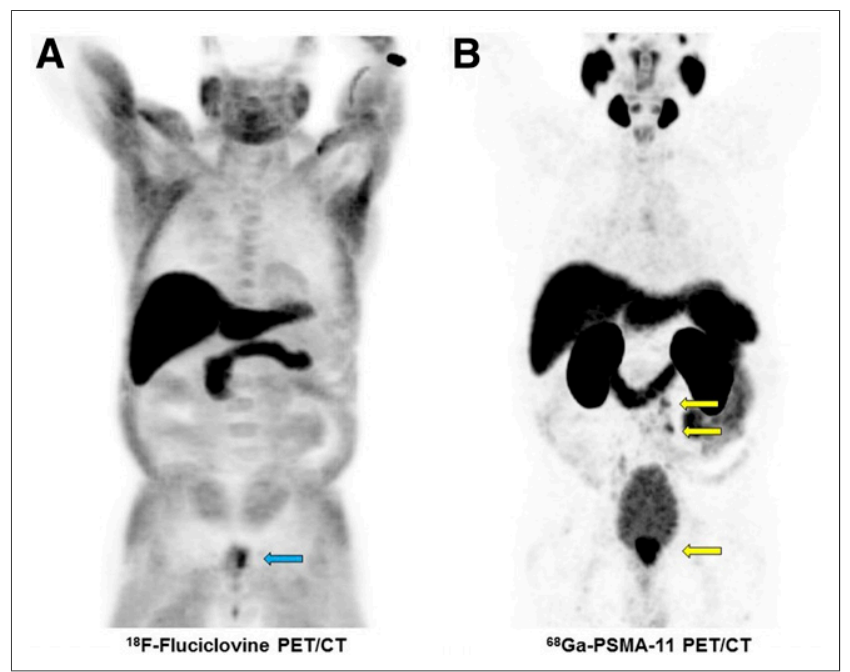

FIGURE 5. ${ }^{18} \mathrm{~F}$-fluciclovine (A) and ${ }^{68} \mathrm{Ga}$-PSMA (B) uptake on PET images of patient with metastatic prostate cancer. Faint uptake of ${ }^{18} \mathrm{~F}$-fluciclovine is seen in left prostate gland (blue arrow), and uptake of ${ }^{68} \mathrm{Ga}-\mathrm{PSMA}$ is seen in prostate gland and extrapelvic lymph nodes (yellow arrows). cancer no longer responding to other treatments. In February 2017, the FDA granted investigational-new-drug clearance allowing for the first clinical trials to begin.

${ }^{177} \mathrm{Lu}$ has ideal physical properties for use in cancer treatment, with a particle range of $1.5 \mathrm{~mm}$ and a half-life of $6.73 \mathrm{~d}$ (7). This short range provides better irradiation of small tumors while sparing the surrounding normal tissue. This treatment is administered via slow intravenous injection. There are essentially no side effects at the time of administration. After injection, radiation safety precautions need to be maintained just as with any other radiotracer excreted via renal clearance. Doses typically range between 3 and $8 \mathrm{GBq}(81-216 \mathrm{mCi})$ per treatment. The number of therapy regimens is generally up to 6 , with a minimum of 6 wk between administrations. Dosimetry imaging can better determine the patient-specific dose. Patient preparation has yet to be finalized because the clinical trials are still ongoing.

\section{RADIOPHARMACEUTICALS FOR NEUROLOGIC PET IMAGING}

Neurologic PET imaging has grown exponentially in recent years. There have been numerous new tracers approved by the FDA, as well as some that have yet to be approved but are currently in clinical trials. These new tracers have expanded the value of PET brain imaging to include the diagnosis of amyloid plaque to assist in evaluating patients with cognitive impairment and dementia.

Alzheimer disease is the most common form of dementia, accounting for $60 \%-80 \%$ of all dementia cases (20). Although investigators have yet to prove the cause of dementia, they believe that the suspected culprits are amyloid plaque and tau tangles within the brain (20). Imaging can be performed with any of the currently FDA-approved cyclotronproduced agents: ${ }^{18} \mathrm{~F}$-florbetapir, ${ }^{18} \mathrm{~F}$-flutametamol, and ${ }^{18} \mathrm{~F}$ florbetaben (21-23). A positive scan result does not in and of itself mean a patient has Alzheimer disease. A positive test result indicates that the patient has amyloid plaque present in the brain, which may make the patient more susceptible to developing Alzheimer disease in the future.

\section{${ }^{18}$ F-Florbetapir}

${ }^{18} \mathrm{~F}-$ florbetapir (Amyvid; Lilly) was the first amyloid imaging radiotracer to receive FDA approval, in 2012 (21). The recommended dose for imaging with ${ }^{18} \mathrm{~F}$-florbetapir is $370 \mathrm{MBq}(10 \mathrm{mCi})$. The dose should be administered through a short intravenous catheter $(<1.5$ in) to minimize dose adherence to the catheter. There is no patient preparation before the study. The uptake time is $30-50 \mathrm{~min}$, followed by a $10-$ min scan (Fig. 6). According to the ${ }^{18} \mathrm{~F}$-florbetapir package insert, a small percentage of patients reported adverse reactions, including flushing, headache, increased blood pressure, nausea, and dizziness. Elimination of the radiopharmaceutical is primarily through the biliary/gastrointestinal tract and a minimal amount excreted via urine. The absorbed radiation dose at the recommended administered dose was $7 \mathrm{mSv}$. The 


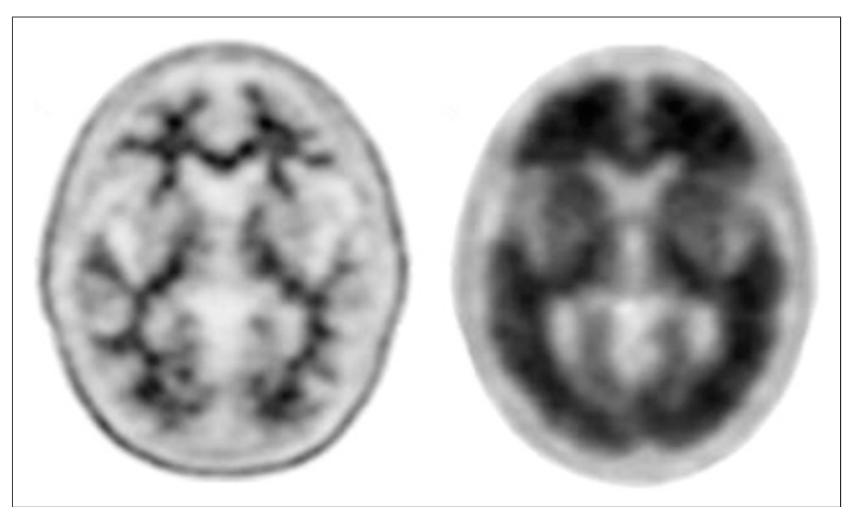

FIGURE 6. Transaxial PET slices demonstrating ${ }^{18} \mathrm{~F}$ florbetapir-negative scan (left) and amyloid-positive scan (right).

maximum intensity of the display scale is set to the brightest region of overall brain uptake.

\section{${ }^{18}$ F-Flutametamol}

${ }^{18} \mathrm{~F}$-flutametamol (Vizamyl; GE Healthcare) received approval in 2013. The recommended dose is $185 \mathrm{MBq}(5 \mathrm{mCi})$ administered via an intravenous bolus injection (22). No patient preparation is required for the study; however, patients should be instructed to remain well hydrated before and after receiving the radiopharmaceutical injection and to void frequently for $24 \mathrm{~h}$ after the study. The 20-min image acquisition begins approximately $90 \mathrm{~min}$ after injection (Fig. 7). According to the ${ }^{18} \mathrm{~F}$-flutemetamol package insert, a small percentage of patients reported adverse reactions, including flushing, headache, increased blood pressure, nausea, and dizziness. Elimination of the radiopharmaceutical is approximately 52\% hepatobiliary and 35\% renal. The absorbed radiation dose at the recommended administered dose was $5.9 \mathrm{mSv}$. When displaying the images, the scale intensity is set to $90 \%$ in the pons region.

\section{${ }^{18}$ F-Florbetaben}

In 2014, the FDA approved a third amyloid imaging agent, ${ }^{18} \mathrm{~F}$-florbetaben (Neuraceq; Piramal). The recommended dose of ${ }^{18} \mathrm{~F}$-florbtaben is $300 \mathrm{MBq}(8.1 \mathrm{mCi})$ injected intravenously (23). There is no patient preparation

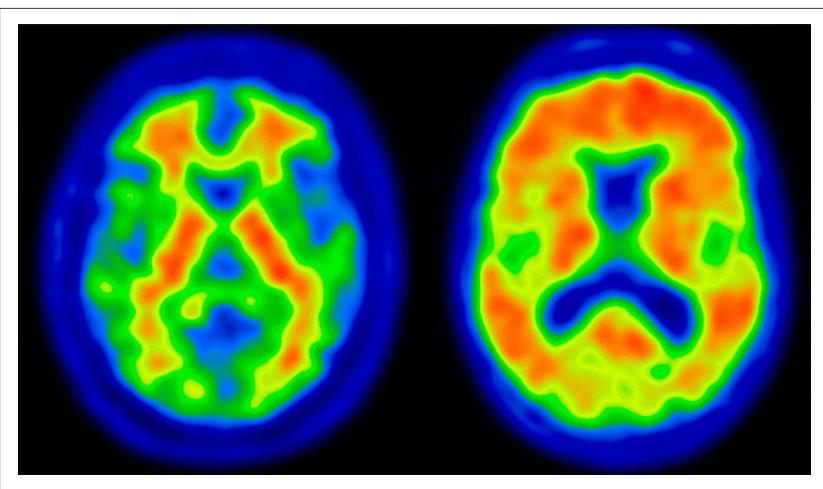

FIGURE 7. Transaxial PET slices demonstrating ${ }^{18} \mathrm{~F}$-flutametamolnegative scan (left) and amyloid-positive scan (right). for the study. A 15- to 20-min scan is performed 45-130 min after injection (Fig. 8). Reported reactions to ${ }^{18} \mathrm{~F}$-florbetaben consist of injection site irritation, erythema, and pain. Primary elimination of the radiopharmaceutical is via the hepatobiliary route, with $30 \%$ of the injected dose excreted in the urine at $12 \mathrm{~h}$. The absorbed radiation dose at the recommended administered dose was $5.8 \mathrm{mSv}$. The white-matter maximum is the reference for images acquired with ${ }^{18} \mathrm{~F}$ florbetaben.

\section{RADIOPHARMACEUTICALS FOR MYOCARDIAL PERFUSION IMAGING}

Every year in the United States, 790,000 people have a heart attack, equating to 1 every $40 \mathrm{~s}$ (24). Coronary artery disease is the primary cause of heart attacks. Common signs and symptoms of a heart attack include pain in the jaw, neck, back, arms, or shoulders; weakness; a feeling of light-headed or faintness; chest pain or discomfort; shortness of breath; and nausea or vomiting. Symptoms may be different for women. However, it is important to note that 1 in 5 heart attacks are silent, with no symptoms experienced.

Most institutions perform nuclear myocardial perfusion imaging using SPECT with ${ }^{99 \mathrm{~m}} \mathrm{Tc}$-labeled agents. Coronary flow reserve imaging, a newer component of cardiac imaging, helps to diagnosis small-vessel disease as well as multivessel and triple-vessel disease. Triple-vessel disease is poorly diagnosed with SPECT imaging. Several SPECT and PET myocardial perfusion imaging and coronary flow reserve radiopharmaceuticals are currently available. A new cardiac PET myocardial perfusion imaging agent is currently under investigation (25).

${ }^{18} \mathrm{~F}$-flurpiridaz is currently being developed by Lantheus Medical Imaging as a PET myocardial perfusion agent. It has been reported to be useful in patients who are difficult to image, including women and obese patients (25). In clinical trials, a 2-d protocol is used. Patients are injected

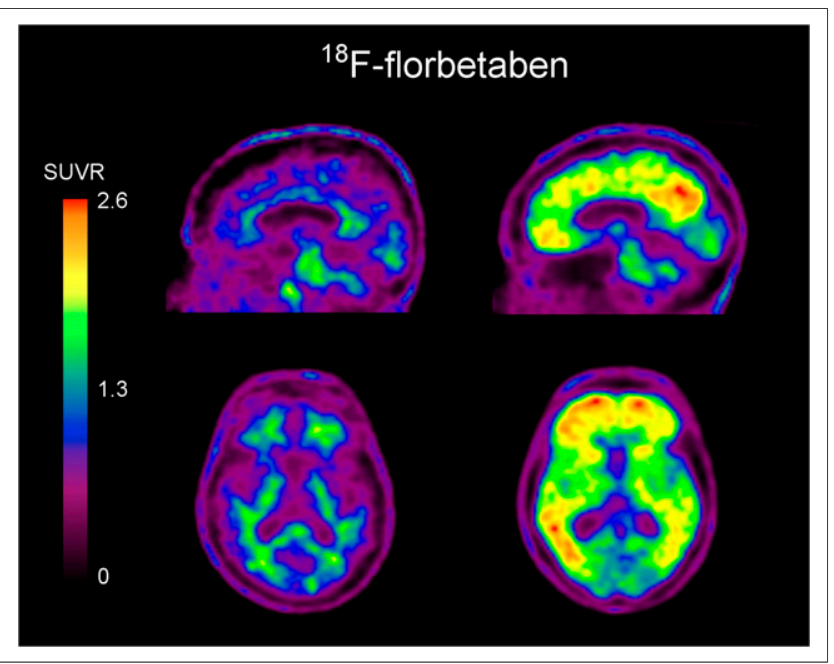

FIGURE 8. Sagittal and transaxial PET slices demonstrating ${ }^{18} \mathrm{~F}$-florbetaben-negative scan (left) and ${ }^{18} \mathrm{~F}$-florbetaben-positive scan (right). SUVR = SUV ratio. 


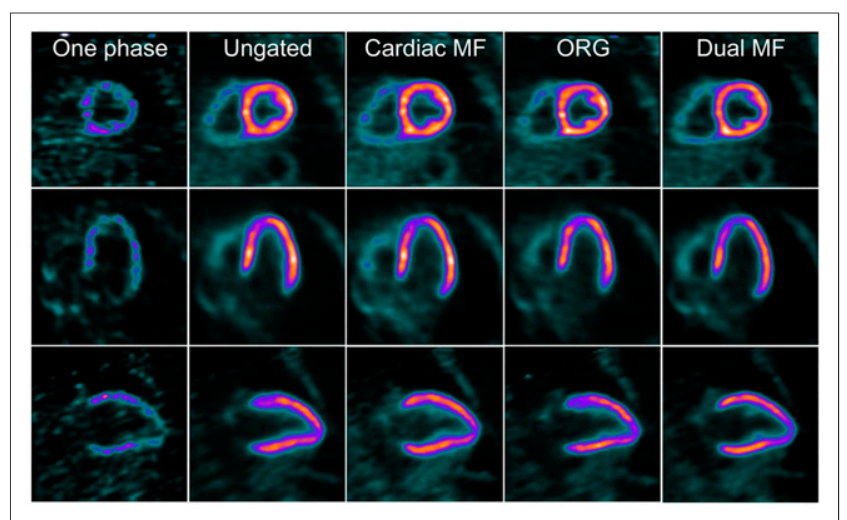

FIGURE 9. Short-axis (top), vertical long-axis (middle), and horizontal long-axis (bottom) PET myocardial perfusion images obtained using ${ }^{18} \mathrm{~F}$-flurpiridaz during various cardiac and respiratory gating. Cardiac MF = cardiac motion frozen, no respiratory gating; $\mathrm{ORG}=$ optimal respiratory gating.

at rest on day 1 and return on day 2 to undergo a stress test (Fig. 9). Since the half-life of ${ }^{18} \mathrm{~F}$ is $110 \mathrm{~min}$, stress testing can be performed with either pharmacologic or exercise stress. Dosimetry results indicate that doses of up to 518 $\mathrm{MBq}(14 \mathrm{mCi})$ provide excellent image quality while maintaining an acceptable effective dose for the patient (26).

\section{CONCLUSION}

Although not all-inclusive, this article does highlight a good cross section of what is occurring in the field of PET diagnostic agents and related therapeutic radiopharmaceuticals. As research progresses, our arsenal of imaging and therapeutic agents will continue to expand. This expansion allows us to stay in the forefront of precision and personalized medicine, giving our patients a multitude of diagnostic and treatment options that provide the highest level of care.

\section{DISCLOSURE}

No potential conflict of interest relevant to this article was reported.

\section{REFERENCES}

1. The neuroendocrine system. Canadian Cancer Society website. http://www.cancer.ca/ en/cancer-information/cancer-type/neuroendocrine/neuroendocrine-tumours/theneuroendocrine-system/?region=on. Accessed April 25, 2019.
2. Oronsky B, Ma PC, Morgensztern D, Carter CA. Nothing but NET: a review of neuroendocrine tumors and carcinomas. Neoplasia. 2017;19:991-1002.

3. Deppen SA, Liu E, Blume JD, et al. Safety and efficacy of ${ }^{68} \mathrm{Ga}$-DOTATATE PET/CT for diagnosis, staging, and treatment management of neuroendocrine tumors. J Nucl Med. 2016;57:708-714.

4. NETSPOT (kit for the preparation of gallium Ga 68 DOTATATE injection) [package insert]. Saluggia (Vc), Italy: Gipharma, S.r.l.; 2016.

5. Hope TA, Bergsland EK, Bozkurt MF, et al. Appropriate use criteria for somatostatin receptor PET imaging in neuroendocrine tumors. J Nucl Med. 201;59:66-74.

6. Lutathera ${ }^{\circledR}$ [product monograph]. Millburn, NJ: Advanced Accelerator Applications USA, Inc.; January 2018.

7. Kam BL, Teunissen JJ, Krenning EP, et al. Lutetium-labelled peptides for therapy of neuroendocrine tumours. Eur J Nucl Med Mol Imaging. 2012;39(suppl 1): S103-S112.

8. Intra-arterial peptide receptor radionuclide therapy (IA-PRRT) using ${ }^{90}$ Y-DOTATOC. Neuroendocrine Tumor Research Foundation website. https://netrf.org/ peptide-receptor-radionuclide-therapy-prrt/. Accessed April 25, 2019.

9. Lutetium Lu 177 DOTATATE [package insert]. Colleretto Giacosa (TO), Italy: Advanced Accelerator Applications USA, Inc; 2018.

10. About prostate cancer. American Cancer Society website. https://www.cancer. org/cancer/prostate-cancer/about.html/. Accessed April 25, 2019.

11. Fluciclovine F 18 [package insert]. Oxford, U.K.: Blue Earth Diagnostics Ltd.; 2016.

12. Parent EE, Schuster DM. Update on ${ }^{18} \mathrm{~F}$-fluciclovine PET for prostate cancer imaging. J Nucl Med. 2018;59:733-739

13. Awwad HM, Geisel J, Obeid R. The role of choline in prostate cancer. Clin Biochem. 2012;45:1548-1553.

14. Choline [package insert]. Noblesville, IN: Zevacor Molecular; 2012.

15. Schillaci O, Calabria F, Tavolozza M, et al. ${ }^{18} \mathrm{~F}$-choline PET/CT physiological distribution and pitfalls in image interpretation: experience in 80 patients with prostate cancer. Nucl Med Commun. 2010;31:39-45.

16. Vali R, Loidl W, Pirich C, Langesteger W, Beheshti M. Imaging of prostate cancer with PET/CT using ${ }^{18}$ F-fluorocholine. Am J Nucl Med Mol Imaging. 2015;5:96108.

17. Jadvar H. Prostate cancer: PET with ${ }^{18} \mathrm{~F}-\mathrm{FDG},{ }^{18} \mathrm{~F}$ - or ${ }^{11} \mathrm{C}$-acetate, and ${ }^{18} \mathrm{~F}$ - or ${ }^{11}$ C-choline. J Nucl Med. 2011;52:81-89.

18. Regula NK, Lubberink M, Jorulf H, Ladjevardi S, Haggman M, Sorensen J. Dynamic imaging of prostate cancer with ${ }^{11} \mathrm{C}$-acetate PET/CT [abstract]. J Nucl Med. 2017;58(suppl 1):662.

19. Fendler WP, Eiber M, Beheshti M, et al. ${ }^{68} \mathrm{Ga}$-PSMA PET/CT: joint EANM and SNMMI procedure guideline for prostate cancer imaging: version 1.0. Eur J Nucl Med Mol Imaging. 2017;44:1014-1024.

20. What is Alzheimer's? Alzheimer's Association website. https://www.alz.org/ alzheimers-dementia/what-is-alzheimers. Accessed April 25, 2019.

21. Florbetapir F.18 [package insert]. Indianapolis, IN: Eli Lily and Company; 2013.

22. Flutemetamol F 18 [package insert]. Arlington Heights, IL: GE Healthcare; 2013.

23. Florbetaben F18 [package insert]. Dulles, VA: Piramal Imaging; 2014.

24. Heart attack. Centers for Disease Control and Prevention website. https://www. cdc.gov/heartdisease/heart_attack.htm. Accessed April 25, 2019.

25. Flurpiridaz F 18. Lantheus Medical Imaging website. http://www.lantheus.com/ pipeline/flurpiridaz-f-18/. Accessed April 25, 2019

26. Maddahi J, Packard R. Flurpiridaz F-18 may expand PET for myocardial perfusion imaging. Imaging Technology News website. https://www.itnonline.com/article/ flurpiridaz-f-18-may-expand-pet-myocardial-perfusion-imaging. Accessed April 25, 2019. 